\title{
The effect of valproic acid on bovine oocyte maturation and early embryonic development in vitro
}

\author{
Haixia Gao $\cdot$ Haidong Bai $\cdot$ Xudong Ao $\cdot$ \\ Rula Sa $\cdot$ Huimin Wang $\cdot$ Zhenfei Wang \\ Yongli Yue $\cdot$ Haiquan Yu
}

Received: 21 January 2013/Accepted: 8 June 2013/Published online: 10 July 2013

(C) Springer Science+Business Media Dordrecht 2013

\begin{abstract}
Our objective is to investigate the effect of valproic acid (VPA), a histone deacetylase inhibitor, on early embryonic development. We studied the effect of VPA on the in vitro maturation of bovine oocytes, and on the development of bovine embryos derived from in vitro fertilization (IVF) or parthenogenesis. Germinal vesicle stage bovine oocytes were cultured with different concentrations of VPA for $24 \mathrm{~h}$; low dose VPA treatment $(0.03$ and $0.3 \mathrm{mM})$ had no effect on oocyte maturation, but 3 and $6 \mathrm{mM}$ VPA significantly decreased maturation rate; when used for IVF or parthenogenesis, VPA-treated oocytes generated significantly lowered blastocyst rate. Oocytes matured in vitro were fertilized or underwent parthenogenetic activation; $6 \mathrm{~h}$ later, they were exposed to VPA for $48 \mathrm{~h}$, and then the cleavage rate, blastocyst rate and mRNA expression levels of transcription factors (Oct4, Nanog, and Cdx2) were assessed. For embryos cultured in $0.3 \mathrm{mM}$ VPA, there was no remarkable change in cleavage rate or blastocyst rate, but the expression of Oct4 and Nanog in blastocysts was significantly increased. For embryos treated with $3.0 \mathrm{mM}$ VPA, the cleavage rate and blastocyst rate were significantly decreased. In conclusion, low dose
\end{abstract}

H. Gao $\cdot$ H. Bai $\cdot$ X. Ao $\cdot$ R. Sa $\cdot$ H. Wang

Z. Wang $\cdot$ Y. Yue $\cdot$ H. Yu $(\bowtie)$

The Key Laboratory of Mammal Reproductive Biology

and Biotechnology, Ministry of Education, Inner

Mongolia University, Hohhot 010021, China

e-mail: haiquan_yu@yahoo.com
VPA has no effect on oocyte maturation but affects subsequent embryonic development. Low dose VPA administration to IVF embryos had no effect on embryonic development, but the expression of several important transcription factors was increased. Treatment of IVF embryos with low dose VPA may improve their development potential.

Keywords Valproic acid - Oocyte maturation . Parthenogenesis $\cdot$ In vitro fertilization

\section{Introduction}

Since 1997, when a cloned sheep named Dolly was created by somatic cell nuclear transfer, various other cloned animals have been produced by using the same method (Lorthongpanich et al. 2010). This technique is of significant importance in basic research and biomedicine; however, there are still some limitations, such as the low developmental rate of cloned embryos, heteroplasia of placentas, high rates of miscarriage and the occurrence of congenital birth defects. It is widely recognized that the main reason for these defects is the insufficient epigenetic reprogramming during early embryogenesis, the underlying mechanisms of which are not yet clear. In recent years, it has been found that some small molecules possess epigenetically regulatory activities that can improve cloning efficiency and the quality of cloned embryos (Wu et al. 2008). The proper screening and characterization of these 
small molecules to determine their function will provide an important basis for improving the somatic cell nuclear transfer technique.

Valproic acid (VPA), a synthetic short chain fatty acid composed of eight carbon atoms, has been used for the treatment of epilepsy since the nineteenth century (Phiel et al. 2001). Mechanistic studies have revealed that VPA is a histone deacetylase inhibitor (HDACi), with the specific inhibiting activity against histone deacetylases (HDACs), thereby promoting the acetylation of cellular histones with little toxicity or side effects (Chen et al. 2011). In support of this, it was found that VPA can be used to treat certain cancers via its inhibitory activity of HDACs (Wang et al. 2011). Furthermore, it was also found that VPA treatment leads to the up-regulation of $p 21 W A F 1 / C I P 1$ and down-regulation of $C d k 2$, with a resultant inhibitory effect on the cell cycle (Ocker and Schneider-Stock 2007). With respect to nerve cell repair, VPA was found to have a protective effect towards damaged nerve cells, being able to increase the survival rate of retinal ganglion cells in rats (Penas et al. 2011). VPA can directly reprogram human fibroblasts into induced pluripotent stem cells without transfection of transcription factors, such as Klf4 and c-myc (Huangfu et al. 2008). Furthermore, it has been found that VPA treatment can increase the expression of pluripotency genes in murine myocytes (Teng et al. 2010) and bovine adipose stem cells (Addison et al. 2011), which indicates that VPA promotes the maintenance of a pluripotent state. VerMilyea et al. found that VPA did not affect the developmental rate of cloned embryos, but delayed embryonic development in mice (VerMilyea et al. 2009); however, Miyoshi et al. (Miyoshi et al. 2010) found that VPA could improve the blastocyst rate in their study on cloning miniature pigs.

To date, research on VPA has mainly focused on the influence of treating donor cells with VPA on the development of cloned embryos and the dynamic change of histone acetylation, with less emphasis on the effect of VPA treated oocytes and embryos in vitro. In this study, using oocyte in vitro maturation, in vitro fertilization (IVF) and parthenogenetic activation, we aimed to investigate the effect of VPA, on bovine oocyte maturation and early embryonic development. The results of this study provide an improved understanding of how VPA influences early embryonic development, with potential applications in the creation of cloned bovine embryos.

\section{Materials and methods}

\section{Reagents}

Oocyte maturation medium (TCM199) was purchased from Gibco-BRL (Gaithersburg, MD, USA). Fetal bovine serum (FBS) was purchased from TBD Biotechnology Development (Tianjin, China). RNAiso Reagent, RT-PCR kit and SYBR green master mix were from TaKaRa (Dalian, China). Caffeine, D-Glucose, D-Lactose, Na-Citrate- $2 \mathrm{H}_{2} \mathrm{O}, \mathrm{Na}_{2} \mathrm{HPO}_{4}$, $\mathrm{K}_{2} \mathrm{HPO}_{4}, \mathrm{NaHCO}_{3}, \mathrm{NaCl}$ and $\mathrm{KCl}$ were purchased from Wako (Osaka, Japan). Unless otherwise mentioned, all other reagents used were purchased from Sigma-Aldrich (St. Louis, MO, USA).

In vitro oocyte collection and maturation

Fresh bovine ovaries were collected from abattoir, placed into sterilized normal saline $\left(35^{\circ} \mathrm{C}\right)$ and brought to our laboratory within $3 \mathrm{~h}$. A $10-\mathrm{mL}$ syringe with an 18 gauge needle was used to aspirate follicles $(2-8 \mathrm{~mm})$ from the ovary surface. Cumulus oocyte complexes (COCs) were gathered from follicular fluid using a stereomicroscope and were transferred into TCM199 (with $0.38 \mathrm{mmol} / \mathrm{L}$ sodium pyruvate, $10 \mu \mathrm{g} / \mathrm{mL}$ FSH, $5 \mu \mathrm{g} / \mathrm{mL}$ LH, $1 \mu \mathrm{g} / \mathrm{mL}$ $17-\beta$ estradiol, $50 \mathrm{U} / \mathrm{mL}$ penicillin, $100 \mu \mathrm{g} / \mathrm{mL}$ streptomycin and $10 \%$ FBS) after washing three to four times. $80-100$ COCs were placed into $1 \mathrm{~mL}$ pre-equilibrated TCM199 overlaid with mineral oil in a well of a 4-well plate. Oocyte maturation was conducted under the conditions of $38.5{ }^{\circ} \mathrm{C}, 5 \% \mathrm{CO}_{2}$ and saturated humidity.

\section{In vitro fertilization}

Frozen breeding bull semen (Livestock Improving Station, Inner Mongolia, China) was thawed in a $37{ }^{\circ} \mathrm{C}$ water bath, and washed twice by centrifugation (5 min at 3,500 rpm) using BO liquid containing $10 \mathrm{mM}$ caffeine. After washing two times by centrifugation, floating sperm were carefully aspirated and incubated in an equal volume of BO with $3 \mathrm{mg} / \mathrm{mL}$ BSA and $8 \mu \mathrm{L} / \mathrm{mL}$ heparin. Sperm was then counted using a microscope. The sperm suspension was aliquoted into $100 \mu \mathrm{L}$ droplets, one of which was added into cultured droplets containing 15-20 mature oocytes. Six to seven hours after fertilization, cumulus 
cells were removed, and the fertilized eggs were placed into $40 \mu \mathrm{L}$ droplets of maturation medium (CR1 plus $0.8 \%$ FBS). Cleavage and blastocyst rate was determined on the 2 nd and 6th to 8th day of culture, respectively.

Parthenogenetic activation

COCs matured in vitro for $22 \mathrm{~h}$ were digested with $0.1 \%$ hyaluronidase to remove cumulus cells. After washing three times with maturation medium, oocytes that had extruded first polar bodies and had even cytoplasm and good morphology were collected for further use. Selected oocytes were placed into the development medium containing $5 \mu \mathrm{M}$ ionomycin for $5 \mathrm{~min}$, and they were then incubated for $6 \mathrm{~h}$ with CR1 medium containing $10 \mu \mathrm{g} / \mathrm{mL}$ 6-dimethylaminopurine, at $38.5{ }^{\circ} \mathrm{C}$, in $5 \% \mathrm{CO}_{2}$ and saturated humidity. Lastly, activated oocytes were washed three times with CR1 medium, and then transferred into $40 \mu \mathrm{L}$ droplets of CR1 medium covered with mineral oil and cultured at $38.5{ }^{\circ} \mathrm{C}$, in $5 \% \mathrm{CO}_{2}$ with saturated humidity. The cleavage and blastocyst rates were examined on the 3nd and 6th to 8th day of culture, respectively.

\section{Feeder layer preparation}

Bovine cumulus cells were used as feeder layers. After in vitro maturation, COCs were digested with $0.1 \%$ hyaluronidase to release cumulus cells. The cumulus cells were collected, washed three times with DMEM medium by centrifugation, and then cultured in DMEM medium containing $10 \%$ FBS. Cells at passage 7-8 were collected by trypsinization and suspended in $10 \%$ FBS-DMEM medium to a density of $1.0 \times 104 / \mathrm{mL}$. Aliquots of $40 \mu \mathrm{L}$ cell suspension were placed under mineral oil in $60 \mathrm{~mm}$ culture dishes. Before embryo culture, the DMEM medium was replaced with CR1 medium.

\section{Real-time quantitative PCR}

Total RNA from embryos in different treatment groups were extracted using TaKaRa RNAiso reagent. In brief, the embryos were lyzed in RNAiso reagent and total RNA was extracted by chloroform, which was then washed using $75 \%$ alcohol and dissolved in nuclease-free water. The concentration and quality of total extracted RNA was then calculated by ultraviolet spectrophotometry. Isolated total RNA was reversetranscribed into cDNA, which was subsequently used for real-time quantitative PCR. The reference gene Gapdh was used to normalize the relative expression levels of the transcription factors, Oct4, Nanog, and $C d x 2$. The respective threshold value for each gene was determined and the relative transcriptional level between groups was calculated using the $2^{-\Delta \Delta c t}$ method. The PCR cycling conditions were as follows: 40 cycles of $4{ }^{\circ} \mathrm{C}$ for $30 \mathrm{~s}, 94{ }^{\circ} \mathrm{C}$ for $5 \mathrm{~s}$, and $60{ }^{\circ} \mathrm{C}$ for $31 \mathrm{~s}$. A total reaction volume of $20 \mu \mathrm{L}$ was used for all PCR reactions. All primer sequences are shown in Table 1. Primers were designed using the bovine mRNA sequences of Oct4, Nanog and Cdx2 obtained from GenBank, using Primer Premier 3.0 software. Primers were synthesized by Dalian TaKaRa Biotechnology Co. Ltd.

\section{Experimental design}

Experiment one: germinal vesicle $(\mathrm{GV})$ stage bovine oocytes were treated with different concentrations $(0$, $0.03,3$ and $6 \mathrm{mM}$ ) of VPA for $24 \mathrm{~h}$ to determine the effect of VPA on the maturation of bovine oocytes. This initial experiment was used to determine what dose of VPA to be used for follow-up studies. Bovine oocytes treated with VPA were subsequently used for studies on its effect the development of embryos produced by IVF or parthenogenetic activation in vitro.

Experiment two: Oocytes matured in vitro were used for IVF. After $6 \mathrm{~h}$ of fertilization, granular cells were removed and the IVF embryos were put into CR1 droplets with different concentrations of VPA and incubated for $48 \mathrm{~h}$. Then, after washing 3 times in normal CR1 medium, the embryos were cultured in normal CR1 medium containing a feeder layer for a further 6 days for the purpose of determining blastocyst rate.

Experiment three: Oocytes matured in vitro were used for parthenogenetic activation. After $6 \mathrm{~h}$ of activation, the parthenogenetic embryos were placed into CR1 droplets with different concentrations of VPA and incubated for $48 \mathrm{~h}$. Then, after washing 3 times in normal CR1 medium, they were cultured in normal CR1 medium containing a feeder layer for 6 days for the purpose of detecting the blastocyst rate. 
Table 1 Primer sequences

\begin{tabular}{llc}
\hline Gene & Primers & Length (bp) \\
\hline Oct4-F & AGTAGGTTGGGTAGAGGGGTATTAG & 184 \\
Oct4-R & AAATTAAAAAAATCTCCTAAAAAAAA & \\
Nanog-F & ATAATGGTTTTGGTGAGATTGGTAG & 161 \\
Nanog-R & ATAAAACTCAACCATACTTAACCCC & \\
CDX2-F & AAGACAAATACCGGGTCGTG & 154 \\
CDX2-R & CTGCGGTTCTGAAACCAAAT & 119 \\
GAPDH & $5^{\prime}$-TTCAACGGCACAGTCAAGG-3' & \\
GAPDH & 5'-ACATACTCAGCACCAGCATCAC-3' $^{\prime}$ & \\
\hline
\end{tabular}

\section{Results}

Effect of VPA treatment on bovine oocyte maturation in vitro and early embryonic development

Bovine GV oocytes were in vitro matured in medium containing 0, 0.03, 0.3, 3 and 6 mM VPA. We found that the maturation rate decreased after $24 \mathrm{~h}$ treatment with increasing concentrations of VPA. Low doses of VPA (0.03 and $0.3 \mathrm{mM})$ had no significant effect on the maturation rate; however, high doses of VPA ( $3 \mathrm{mM}$ and $6 \mathrm{mM}$ ) significantly decreased the maturation rate $(p<0.01$, Table 2$)$. Treatment of oocyte with $6 \mathrm{mM}$ VPA also greatly reduced cleavage rate and abolished the development to blastocyst after IVF, indicating its toxic effect on bovine oocytes at this concentration (Table 3). Furthermore, the majority of bovine oocytes died after the application of $12 \mathrm{mM}$ VPA. Based on the above results, follow-up experiments were conducted using 0.3 and $3 \mathrm{mM}$ VPA.

Effect of VPA treatment of bovine oocytes on early embryonic development

The oocytes in vitro-matured in the presence of $0,0.3$ and $3 \mathrm{mM}$ VPA were subjected to IVF. VPA treatment significantly lowered the cleavage and blastocyst rates. Specifically, the cleavage rate of the $0.3 \mathrm{mM}$ VPA-treated group was significantly lower than that of control group $(p<0.05)$, as was the blastocyst rate $(p<0.01)$. Both the cleavage rate and blastocyst rate of $3 \mathrm{mM}$ VPA-treated oocytes were significantly lower than those of control group ( $p<0.01$, Table 4 ).

The $0,0.3$ and $3 \mathrm{mM}$ VPA-treated oocytes were also subjected to parthenogenetic activation, and the cleavage and blastocyst rates were examined.
Table 2 Effect of VPA treatment on the maturation rate of bovine oocytes-dose response analysis

\begin{tabular}{llll}
\hline $\begin{array}{l}\text { Concentration } \\
(\mathrm{mM})\end{array}$ & $\begin{array}{l}\text { No. of } \\
\text { oocytes }\end{array}$ & $\begin{array}{l}\text { No. of } \\
\text { matured } \\
\text { oocytes }\end{array}$ & $\begin{array}{l}\text { Maturation rate } \\
(\%)\end{array}$ \\
\hline 0.00 & 435 & 327 & $75.17 \% \pm 0.005^{\mathrm{a}}$ \\
0.03 & 323 & 240 & $74.23 \% \pm 0.004^{\mathrm{a}}$ \\
0.30 & 243 & 180 & $74.15 \% \pm 0.004^{\mathrm{a}}$ \\
3.00 & 361 & 210 & $58.17 \% \pm 0.007^{\mathrm{b}}$ \\
6.00 & 338 & 152 & $45.00 \% \pm 0.048^{\mathrm{b}}$ \\
\hline
\end{tabular}

Values with different superscripts in the same column differ significantly $(p<0.05)$. Data are means from three individual experiments

Table 3 Effect of treating GV oocytes with $6.00 \mathrm{mM}$ VPA for $24 \mathrm{~h}$ on cleavage rate of IVF embryos

\begin{tabular}{llll}
\hline Treatment & $\begin{array}{l}\text { No. of } \\
\text { oocytes }\end{array}$ & $\begin{array}{l}\text { No. of } \\
\text { cleavage }\end{array}$ & Cleavage rate (\%) \\
\hline $0 \mathrm{mM} \mathrm{24} \mathrm{h}$ & 415 & 263 & $63.21 \% \pm 0.010^{\mathrm{a}}$ \\
$6 \mathrm{mM} \mathrm{24} \mathrm{h}$ & 445 & 124 & $28.41 \% \pm 0.083^{\mathrm{b}}$ \\
\hline
\end{tabular}

Values with different superscripts in the same column differ significantly $(p<0.05)$. Data are means from three individual experiments

Compared with that of control group, the cleavage rate of the $0.3 \mathrm{mM}$ VPA-treated group was not significantly changed, but the blastocyst rate was significantly decreased $(p<0.01)$. Both the cleavage rate and the blastocyst rate of the $3 \mathrm{mM}$ VPA-treated group were significantly lower than those of control group $(p<0.01$, Table 5).

Effect of $48 \mathrm{~h}$ VPA treatment on bovine early embryonic development

Bovine embryos derived from IVF were treated by VPA for $48 \mathrm{~h}$, and then cleavage and blastocyst rates 
Table 4 Effect of treating GV oocytes with VPA for $24 \mathrm{~h}$ on embryonic development after in vitro fertilization

\begin{tabular}{llllrr}
\hline Concentration $(\mathrm{mM})$ & No. of oocytes & No. of cleavage & Cleavage rate $(\%)$ & No. of blastocysts & Blastocyst rate $(\%)$ \\
\hline 0.00 & 505 & 325 & $64.36 \% \pm 0.010^{\mathrm{a}}$ & 112 & $34.46 \% \pm 0.005^{\mathrm{a}}$ \\
0.30 & 440 & 255 & $57.95 \% \pm 0.011^{\mathrm{b}}$ & 34 & $13.33 \% \pm 0.006^{\mathrm{b}}$ \\
3.00 & 488 & 182 & $37.30 \% \pm 0.021^{\mathrm{b}}$ & 13 & $7.20 \% \pm 0.004^{\mathrm{b}}$ \\
\hline
\end{tabular}

Values with different superscripts in the same column differ significantly $(p<0.05)$. Data are means from three individual experiments

Table 5 Effect of treating GV oocytes with VPA for $24 \mathrm{~h}$ on embryonic development after parthenogenetic activation

\begin{tabular}{llllll}
\hline Concentration $(\mathrm{mM})$ & No. of oocytes & No. of cleavage & Cleavage rate $(\%)$ & No. of blastocysts & Blastocyst rate $(\%)$ \\
\hline 0.00 & 244 & 223 & $91.39 \% \pm 0.027^{\mathrm{a}}$ & 85 & $38.12 \% \pm 0.013^{\mathrm{a}}$ \\
0.30 & 154 & 132 & $85.71 \% \pm 0.016^{\mathrm{a}}$ & 20 & $15.15 \% \pm 0.002^{\mathrm{b}}$ \\
3.00 & 275 & 149 & $54.44 \% \pm 0.011^{\mathrm{b}}$ & 16 & $5.56 \% \pm 0.02^{\mathrm{b}}$ \\
\hline
\end{tabular}

Values with different superscripts in the same column differ significantly $(p<0.05)$. Data are means from three individual experiments

Table 6 Effect of VPA treatment after in vitro fertilization on embryonic development

\begin{tabular}{llllrr}
\hline Concentration $(\mathrm{mM})$ & No. of oocytes & No. of cleavage & Cleavage rate $(\%)$ & No. of blastocysts & Blastocyst rate $(\%)$ \\
\hline 0.00 & 210 & 164 & $78.10 \% \pm 0.006^{\mathrm{a}}$ & 84 & $51.21 \% \pm 0.003^{\mathrm{a}}$ \\
0.30 & 273 & 206 & $75.46 \% \pm 0.007^{\mathrm{a}}$ & 105 & $50.97 \% \pm 0.017^{\mathrm{a}}$ \\
3.00 & 146 & $56.81 \% \pm 0.008^{\mathrm{b}}$ & 26 & $17.81 \% \pm 0.005^{\mathrm{b}}$ \\
\hline
\end{tabular}

Values with different superscripts in the same column differ significantly $(p<0.05)$. Data are means from three individual experiments

were assessed. 0.3 mM VPA treatment had little effect on the cleavage rate and blastocyst rate; however, $3 \mathrm{mM}$ VPA treatment significantly decreased cleavage rate and blastocyst rate $(p<0.01$, Table 6).

Bovine embryos obtained by parthenogenetic activation were also treated by VPA for $48 \mathrm{~h}$, and then cleavage and blastocyst rates were assessed. $0.3 \mathrm{mM}$ VPA treatment resulted in a lower cleavage rate and a slightly higher blastocyst rate than control group. $3 \mathrm{mM}$ VPA treatment generated a significantly negative effect on both cleavage rate and blastocyst rate $(p<0.01$, Table 7).

\section{Effect of VPA treatment on Oct4, Nanog and Cdx2 mRNA expression level}

The expression levels of Oct4, Nanog and $C d x 2$ mRNA were assessed by real-time quantitative PCR in blastocyst after IVF embryos were treated with VPA for $48 \mathrm{~h}$ (Fig. 1). Both 0.3 and $3 \mathrm{mM}$ VPA treatment significantly increased the expression levels of Oct4 and Nanog. $3 \mathrm{mM}$ VPA treatment also significantly increased the expression level of $C d x 2(p<0.05)$.

\section{Discussion}

The effect of VPA on epigenetic reprogramming has attracted interest amongst researchers who specialize in cloning animals. It has been shown that the cloning efficiency in pigs could be improved by VPA treatment (Huang et al. 2011; Miyoshi et al. 2010); however, experiments in cloned mouse embryos did not show the same beneficial effect (Ono et al. 2010; Chen et al. 2011). The low cloning efficiency associated with somatic cell nuclear transfer might be related to the low efficiency of in vitro oocyte maturation and embryonic development. This study aimed to explore the effect of VPA on oocyte maturation and the early 
Table 7 Effect of VPA treatment after parthenogenetic activation on embryonic development

\begin{tabular}{llllrr}
\hline Concentration $(\mathrm{mM})$ & No. of oocytes & No. of cleavage & Cleavage rate $(\%)$ & No. of blastocysts & Blastocyst rate $(\%)$ \\
\hline 0.00 & 291 & 267 & $91.75 \% \pm 0.016^{\mathrm{a}}$ & 152 & $56.93 \% \pm 0.007^{\mathrm{a}}$ \\
0.30 & 261 & 230 & $88.12 \% \pm 0.019^{\mathrm{a}}$ & 133 & $57.83 \% \pm 0.022^{\mathrm{a}}$ \\
3.00 & 291 & 229 & $78.70 \% \pm 0.016^{\mathrm{b}}$ & 40 & $17.47 \% \pm 0.015^{\mathrm{b}}$ \\
\hline
\end{tabular}

Values with different superscripts in the same column differ significantly $(p<0.05)$. Data are means from three individual experiments
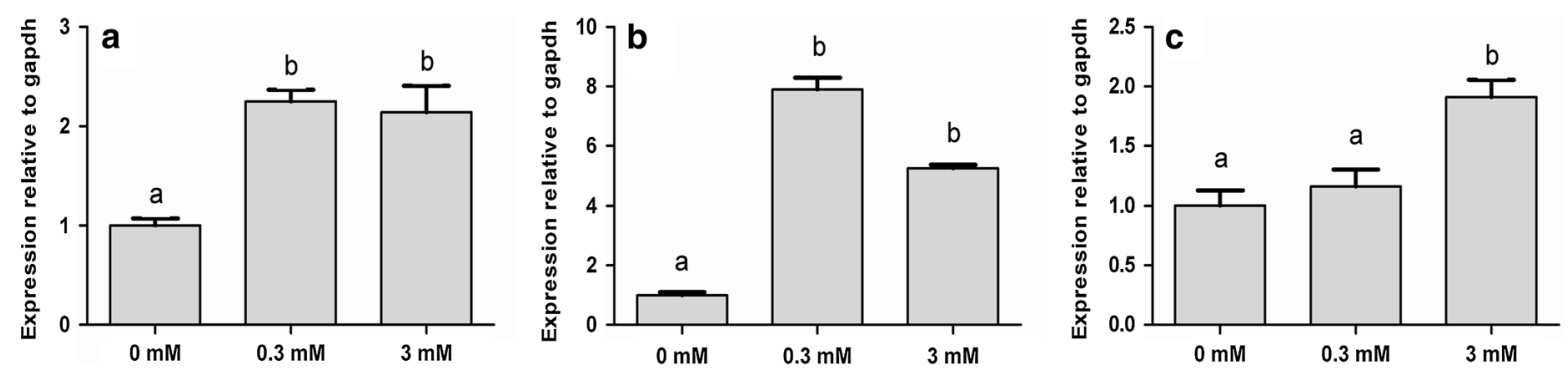

Fig. 1 Effect of treating IVF embryos with VPA on the mRNA expression levels of Oct4 (a), Nanog (b) and Cdx2 (c) in bovine blastocysts

development of embryos obtained by IVF and parthenogenetic activation.

Low dose $(0.3 \mathrm{mM})$ VPA had no effect on the maturation of bovine oocytes, while $3 \mathrm{mM}$ VPA decreased the maturation rate but did not influence oocyte survival. The higher doses of VPA we tested were found to have toxic effects on bovine oocytes and were considered unsuitable for further investigation. The toxic effect of VPA at a higher dose $(6 \mathrm{mM})$ is in agreement with the findings of Wang et al. (2006), who found that a high level of histone acetylation inhibits GV breakdown, and that ordered histone deacetylation is necessary for bovine oocyte to resume meiosis. This same effect might also explain why $3 \mathrm{mM}$ VPA decreased the oocyte maturation rate. These initial investigations established that bovine oocytes can be safely treated with $0.3 \mathrm{mM}$ VPA, and to a slightly lesser extent, with $3 \mathrm{mM}$ VPA. However, exposure of embryos obtained by IVF or parthenogenetic activation to VPA indicated that this compound could negatively affect cleavage rate and blastocyst rate even at concentrations of 0.3 and $3 \mathrm{mM}$. Our data indicate that low dose $(0.3 \mathrm{mM})$ VPA treatment of the bovine embryos does not affect their development; however high dose $(3 \mathrm{mM})$ of VPA did have a negative effect. Interestingly, the effect of VPA treatment was more pronounced when maturing bovine oocytes were exposed to this compound, whereas fertilized oocytes exposed to VPA were relatively less affected.

With regards to the toxic effects of VPA, it has been reported that VPA can delay the cell cycle (Phiel et al. 2001) and embryonic development of mice (VerMilyea et al. 2009), which might explain the effect of $6 \mathrm{mM}$ VPA on bovine oocytes. The death of granular cells could also lead to the delayed development of oocytes. Our data clearly established that exposure to a high concentration of VPA has a strong negative effect on oocyte maturation. Moreover, the negative effect of VPA on bovine oocytes extended to further development. These data indicate that VPA has a long-lasting effect, which does not dissipate after the withdrawal of the compound.

Previous studies have used different does of VPA. Miyoshi et al. (2010) reported that 4 mM VPA could improve blastocyst rate in cloned miniature pigs, while $1 \mathrm{mM}$ VPA was effective in increasing blastocyst rate and embryonic developmental rate in mice (CostaBorges et al. 2010). Moreover, Xu et al. (2012) showed that $4 \mathrm{mM}$ VPA treatment could significantly improve both cleavage and blastocyst rates of bovine somatic cell nuclear transfer embryos, whereas Huang et al. increased cloning efficiency of Landrace pigs with a dose of $1 \mathrm{mM}$. In our study, a dose of $0.3 \mathrm{mM}$ 
VPA was safely used, thus it seems that VPA acts in a relatively wide concentration range and may have different optimal concentrations in different species.

In order to further understand the mechanisms underlying the effect of VPA, we studied the expression level of three important embryonic transcription factors, Oct4, Nanog and $C d x 2.0 .3$ and $3 \mathrm{mM}$ VPA treatment was able to increase the expression levels of both Oct4 and Nanog in blastocysts obtained by IVF. These data are in agreement with the effect of VPA on Oct4 and Nanog expression in bovine adipose stem cells (Huangfu et al. 2008), and Oct4 expression in murine myocytes (Teng et al. 2010). Oct4 is a member of the POU transcription factor family and is known to be an important regulatory factor during early embryonic development, particularly in the pre-implantation embryos (Ovitt and Scholer 1998). Following Oct4 knock-down, embryonic stem cells differentiate to trophectoderm-like cells (Hay et al. 2004). Similarly, Oct $4^{-1-}$ embryos do not form any normal blastocysts (Rosner et al. 1990). Niwa et al. (Niwa et al. 2000) stated that the effect of Oct4 depended on its concentration within nucleus, as such the VPAinduced increase in Oct4 expression we observed could be one reason for the improved quality of VPAexposed embryos. Nanog is a transcription factor expressed in the inner cell mass (ICM), archaeocytes and embryonic stem cells (Mitsui et al. 2003). It has been shown that an ICM lacking Nanog does not generate any ectoderm, but only endoderm-like cells (Mitsui et al. 2003). When lacking Nanog expression, embryonic stem cells lost their pluripotent capacity and differentiated into extraembryonic endoderm, leading to the conclusion that Nanog plays a key role in maintaining embryonic stem cell pluripotency within the ICM (Dietrich and Hiiragi 2007). We observed a VPA-induced increase in Nanog expression, which we interpret to indicate that VPA treatment can improve the quality of blastocysts.

The third gene we studied was $C d x 2$ (Caudal type homeobox transcription factor 2), which is a nuclear transcription factor specifically expressed in the trophectoderm of blastocysts. Heterozygous $C d x 2$ mutant mice have homeotic transformation defects, while homozygous $C d x 2$ mutant mice die before implantation, demonstrating that $C d x 2$ plays an essential role in early embryonic development (Chawengsaksophak et al. 2004). In this study, in embryos derived from IVF, $0.3 \mathrm{mM}$ VPA had a marginal effect on $C d x 2$ expression whereas it was significantly increased by $3 \mathrm{mM}$ VPA. Given the importance of $C d x 2$ in early embryonic development, the inductive effect of VPA on $C d x 2$ expression may be beneficial to the embryonic development after implantation. It can be seen that treatment of IVF embryos with 0.3 and $3 \mathrm{mM}$ VPA both significantly increased the expression of the ICM marker genes Oct4 and Nanog, and $3 \mathrm{mM}$ VPA treatment also significantly increased the expression of the trophectoderm marker gene $C d x 2$. We thus hypothesize that treatment of IVF embryos with 0.3 and $3 \mathrm{mM}$ VPA might be beneficial for the development of ICM at blastocyst stage, and $3 \mathrm{mM}$ VPA treatment also beneficial for the growth of trophectoderm cells.

Our data suggest that VPA treatment has a dosedependent effect on bovine oocyte maturation, with high doses of VPA seriously inhibiting the development of embryos derived from IVF and parthenogenetic activation. Differently, treatment of IVF and parthenogenetic embryos with VPA did not improve their developmental rates, but increased the expression of transcription factors important for early embryogenesis. To date, VPA has been shown to have beneficial effects in the cloning process for pigs and mice. Our data from bovine embryos indicate that VPA treatment can improve the expression of transcription factors important for early embryogenesis, suggesting that VPA may have a beneficial effect in this species. This study provides an important foundation for future studies using VPA treatment to facilitate the creation of cloned bovine embryos.

Acknowledgments This work was supported by a grant from Major Program of the Inner Mongolia Natural Science Foundation (No. 2012ZD04), a grant from the National Key Basic Research Program of China (No. 2010CB134410) and a grant from National Natural Science Foundation of China (No. 30860185) to Haiquan $\mathrm{Yu}$.

\section{References}

Addison MK, Coley LW, Gentry GT, Godke RA, Bondioli KR (2011) 209 epigenetic modification with zebularine and valproic acid and expression of pluripotency genes in bovine adipose stem cells. Reprod Fertil Dev. 24:216-217. doi:http://dx.doi.org/10.1071/RDv24n1Ab209

Chawengsaksophak K, de Graaff W, Rossant J, Deschamps J, Beck F (2004) Cdx2 is essential for axial elongation in mouse development. Proc Natl Acad Sci USA 101:7641-7645. doi:10.1073/pnas.0401654101 
Chen X, Wong JY, Wong P, Radany EH (2011) Low-dose valproic acid enhances radiosensitivity of prostate cancer through acetylated p53-dependent modulation of mitochondrial membrane potential and apoptosis. Mol Cancer Res 9:448-461. doi:1541-7786.MCR-10-0471

Costa-Borges N, Santalo J, Ibanez E (2010) Comparison between the effects of valproic acid and trichostatin A on the in vitro development, blastocyst quality, and full-term development of mouse somatic cell nuclear transfer embryos. Cell Reprogram 12:437-446. doi:10.1089/cell. 2009.0108

Dietrich JE, Hiiragi T (2007) Stochastic patterning in the mouse pre-implantation embryo. Development 134:4219-4231. doi: $10.1242 / \mathrm{dev} .003798$

Hay DC, Sutherland L, Clark J, Burdon T (2004) Oct-4 knockdown induces similar patterns of endoderm and trophoblast differentiation markers in human and mouse embryonic stem cells. Stem Cells 22:225-235. doi:10.1634/stemcells. 22-2-225

Huang Y, Tang X, Xie W, Zhou Y, Li D, Yao C, Zhu J, Lai L, Ouyang H, Pang D (2011) Histone deacetylase inhibitor significantly improved the cloning efficiency of porcine somatic cell nuclear transfer embryos. Cell Reprogram 13:513-520. doi:10.1089/cell.2011.0032

Huangfu D, Osafune K, Maehr R, Guo W, Eijkelenboom A, Chen S, Muhlestein W, Melton DA (2008) Induction of pluripotent stem cells from primary human fibroblasts with only Oct4 and Sox2. Nat Biotechnol 26:1269-1275. doi: nbt. 1502

Lorthongpanich C, Solter D, Lim CY (2010) Nuclear reprogramming in zygotes. Int J Dev Biol 54:1631-1640. doi: $103201 \mathrm{cl}$

Mitsui K, Tokuzawa Y, Itoh H, Segawa K, Murakami M, Takahashi K, Maruyama M, Maeda M, Yamanaka S (2003) The homeoprotein Nanog is required for maintenance of pluripotency in mouse epiblast and ES cells. Cell 113: 631-642. doi:S0092867403003933

Miyoshi K, Mori H, Mizobe Y, Akasaka E, Ozawa A, Yoshida M, Sato M (2010) Valproic acid enhances in vitro development and Oct-3/4 expression of miniature pig somatic cell nuclear transfer embryos. Cell Reprogram 12:67-74. doi:10.1089/cell.2009.0032

Niwa H, Miyazaki J, Smith AG (2000) Quantitative expression of Oct-3/4 defines differentiation, dedifferentiation or selfrenewal of ES cells. Nat Genet 24:372-376. doi:10.1038/ 74199

Ocker M, Schneider-Stock R (2007) Histone deacetylase inhibitors: signalling towards p21cip1/waf1. Int J Biochem Cell Biol 39:1367-1374. doi:S1357-2725(07)00079-9
Ono T, Li C, Mizutani E, Terashita Y, Yamagata K, Wakayama T (2010) Inhibition of class IIb histone deacetylase significantly improves cloning efficiency in mice. Biol Reprod 83:929-937. doi:biolreprod.110.085282

Ovitt CE, Scholer HR (1998) The molecular biology of Oct-4 in the early mouse embryo. Mol Hum Reprod 4:1021-1031

Penas C, Verdu E, Asensio-Pinilla E, Guzman-Lenis MS, Herrando-Grabulosa M, Navarro X, Casas C (2011) Valproate reduces $\mathrm{CHOP}$ levels and preserves oligodendrocytes and axons after spinal cord injury. Neuroscience 178:33-44. doi: S0306-4522(11)00035-2

Phiel CJ, Zhang F, Huang EY, Guenther MG, Lazar MA, Klein PS (2001) Histone deacetylase is a direct target of valproic acid, a potent anticonvulsant, mood stabilizer, and teratogen. J Biol Chem 276:36734-36741. doi:10.1074/jbc. M101287200

Rosner MH, Vigano MA, Ozato K, Timmons PM, Poirier F, Rigby PW, Staudt LM (1990) A POU-domain transcription factor in early stem cells and germ cells of the mammalian embryo. Nature 345:686-692. doi:10.1038/345686a0

Teng HF, Kuo YL, Loo MR, Li CL, Chu TW, Suo H, Liu HS, Lin KH, Chen SL (2010) Valproic acid enhances Oct4 promoter activity in myogenic cells. J Cell Biochem 110:995-1004. doi:10.1002/jcb.22613

VerMilyea MD, O’Neill LP, Turner BM (2009) Transcriptionindependent heritability of induced histone modifications in the mouse preimplantation embryo. PLoS ONE 4:e6086. doi:10.1371/journal.pone.0006086

Wang Q, Yin S, Ai JS, Liang CG, Hou Y, Chen DY, Schatten H, Sun QY (2006) Histone deacetylation is required for orderly meiosis. Cell Cycle 5:766-774. doi:2627

Wang AH, Wei L, Chen L, Zhao SQ, Wu WL, Shen ZX, Li JM (2011) Synergistic effect of bortezomib and valproic acid treatment on the proliferation and apoptosis of acute myeloid leukemia and myelodysplastic syndrome cells. Ann Hematol 90:917-931. doi:10.1007/s00277-011-1175-6

Wu X, Li Y, Li GP, Yang D, Yue YL, Wang LL, Li KH, Xin PH, Bou S, Yu HQ (2008) Trichostatin a improved epigenetic modifications of transfected cells but did not improve subsequent cloned embryo development. Animal Biotechnol 19:211-224. doi:10.1080/10495390802271482

Xu WB, Wang YS, Li YY, Wang LJ, Xiong XR, Su JM, Zhang $Y$ (2012) Valproic acid improves the in vitro development competence of bovine somatic cell nuclear transfer embryos. Cell Reprogram 14:138-145. doi:10.1089/cell. 2011.0084 